\title{
Title: Low Cardiorespiratory Fitness is an Independent Predictor of Metabolic Syndrome in Young Korean Adults
}

\author{
Running Title: Fitness and Insulin Resistance
}

Hyun-Sik Kang ${ }^{1}$, PhD, Soo-Hyun Park ${ }^{1}$, PhD, Shin-Uk Kim², PhD

\footnotetext{
${ }^{1}$ School of Sport Science, Sungkyunkwan University, Suwon, Republic of Korea ${ }^{4}$ Interdisciplinary Program in Bioinformatics, Seoul National University, Seoul, Republic of Korea
}

\author{
Corresponding author: \\ Hyun-Sik Kang, PhD \\ School of Sport Science \\ Sungkyunkwan University, \\ 300 Chenchen-dong, Jangan-Gu \\ Suwon, Republic of Korea, 440-746 \\ Phone: +82-31-299-6911 \\ Fax: +82-31-299-6929 \\ E-mail: hkang@skku.edu
}

Word count of Manuscript: 2397

Number of References: 29

Number of Table: 1

Number of Figure: 1 


\section{ABSTRACT}

Objective: To investigate the relationship between cardio/respiratory fitness (CRF) and metabolic syndrome (MS) in young Korean men. Design: In a cross-sectional study, we

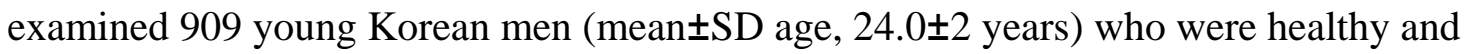
not taking any medications affecting blood pressure, glucose, or lipids concentrations. Body fatness, resting blood pressures, and fasting blood levels of lipids, glucose, and insulin were measured with our standardized laboratory protocols. CRF was quantified as the maximum volume of minute oxygen consumption measured during a graded treadmill test. Metabolic syndrome was defined with the National Cholesterol Education Program Adult Treatment Panel III criteria and a modified cut-off value of waist circumference from the Asia-Pacific Perspective: Redefining Obesity and its Treatment. Results: Group analyses showed significant and inverse dose-response trends between the metabolic syndrome markers and CRF levels such that men with high and moderate CRF levels had more favorable profiles in body fatness, resting blood pressures, mean values in fasting lipids, glucose, and insulin, and homeostasis model of assessment-insulin resistance than men with low CRF level. After adjusting for several potential confounders such as age, smoking, and body fatness variables, the low and moderate CRF groups had odds of 4.64 (95\% CI, 2.00 to 10.79) and 2.57 (95\% CI, 1.04 to 6.34) for having metabolic syndrome than the high CRF group. Conclusion: These findings suggest that low CRF is a significant and independent risk factor for metabolic syndrome in young Korean men.

Key words: Obesity, Physical Fitness, Dietary Intakes, Health Outcomes, Epidemiology. 


\section{INTRODUCTION}

Cardiovascular disease (CVD) is the leading cause of death in both developed and developing countries around the world (1). Metabolic syndrome, the clustering of insulin resistance, dyslipidemia, and hypertension, is known to be associated with the development of type 2 diabetes (2), CVD $(3,4,5)$, and mortality (6). Although the exact etiology of metabolic syndrome is uncertain, both genetic and lifestyle factors are likely to be involved in its development (7).

With the overall Korean lifestyle becoming more like the western one, low physical activity along with a high-fat diet is becoming more prevalent in South Korea (8). Industrialization and the rapid growth of the technology sector have led to a decrease in physical activity including exercise and thus cardio/respiratory fitness (CRF). The overall prevalence of obesity has been substantially increased. All those lifestyle factors appear to contribute to dramatic increases in complications such as metabolic syndrome. In the Korean National Health Examination and Nutrition Survey data from 1998 and $2001(8,9)$, the national prevalence of metabolic syndrome in men aged 20 years or older according to the National Cholesterol Education Program Adult Treatment Panel III (NCEP-ATP III) (10) definition increased from 15.0\% in 1998 to $17.4 \%$ in 2001 . In addition, Koreans appear to be more prone to the development of insulin resistance, a key component of the metabolic syndrome, due to the impairment of early-phase insulin secretion capacity (11).

Substantial evidence supports the use of CRF and physical activity as powerful predictors of health outcomes, including metabolic syndrome, among western populations (2, 12-17). However, no such studies have been conducted in Asians 
including Koreans. Thus, given that CRF is a modifiable and independent risk factor for metabolic syndrome, the purpose of this study was to investigate whether low CRF is a risk factor for the development of metabolic syndrome independent of body fatness in young Korean men.

\section{METHODS}

\section{Subjects and Definition of Metabolic Syndrome}

Subjects for this study included 909 male student volunteers who registered in "health and fitness lectures” at our institute during the interval between 2006 and 2007. All volunteers underwent a comprehensive health and fitness screening at our laboratory. The average rates for the screening were 95\% and 96\% in the 2006 and 2007 semesters, respectively.

The Institutional Research Ethnics Review Committee reviewed and approved the study, and after receiving written informed consent from each participant, a clinical evaluation was performed. The examinations included a standardized health questionnaire, anthropometry and body composition, resting BP and electrocardiogram, fasting blood chemistry, and a maximal graded exercise test on a motor-driven treadmill. Height and body mass were recorded using a stadiometer attached to a scale (Jenix, Seoul, Korea). Waist circumference measurements were made using a cloth tape measure at the level of the umbilicus. Hip girth was measured as the horizontal circumference at the broadest part of the lower body, usually at the level of the trochanters. Percent body fat was assessed using bioelectrical impedance analysis following the procedures 
recommended by the American College of Sports Medicine (ACSM) (18). All procedures were administrated by trained technicians who followed standardized protocols.

Fasting glucose, total cholesterol (TC), triglycerides (TG), and high-density lipoprotein cholesterol (HDLC) concentrations were measured in duplicate using the Ektachem DT-60 II analyzer (Johnson \& Johnson Clinical Diagnostics, Inc., Rochester, NY, USA). Fasting insulin concentrations were also measured in duplicate using a commercially-available ELISA immunoassay kit (Catalog \#: 80-INSHU-E10, ALPCO Diagnostics, Salem, NH, USA). The index of insulin resistance was assessed by using the homoeostasis model of assessment-insulin resistance (HOMA-IR), as HOMA-IR = [fasting insulin $(\mu \mathrm{U} / \mathrm{ml}) \times$ fasting glucose $(\mathrm{mM})] / 22.5(19)$.

The metabolic syndrome was defined according to the NCEP-ATP III (10) except for abdominal obesity, for which we used the cut-off value of waist circumference from the Asian-Pacific Perspective: Redefining Obesity and its Treatment (20). Thus, those who had three or more of the following attributes were considered to have metabolic syndrome: (i) high waist circumference of $\geq 90 \mathrm{~cm}$, (ii) elevated resting BP of $\geq 130 / 85$ mmHg or currently taking antihypertensive medication, (iii) elevated TG of $\geq 150 \mathrm{mg} / \mathrm{dL}$, (iv) low HDLC of $<50 \mathrm{mg} / \mathrm{dL}$, and (v) elevated fasting glucose of $\geq 110 \mathrm{mg} / \mathrm{dL}$.

\section{Maximal Treadmill Test for the $\mathrm{VO}_{2}$ max measurement}

CRF was measured on a motor-driven treadmill using the ACSM guideline (18). The following criteria were used to confirm $\mathrm{VO}_{2}$ max achievement; (i) a leveling off of the $\mathrm{VO}_{2}$, (ii) rate of perceived exertion (RPE) greater than 17 for the original category scale, (iii) volitional exertion, and (iv) achievement of aged-predicted maximal heart rate. Students were encouraged to exercise to exhaustion during the test. 


\section{Statistical Analyses}

In the present analyses, we assigned individuals to low (lowest third), moderate (middle third), and high (top third) CRF tertiles based on age-adjusted $\mathrm{VO}_{2}$ max values. Trends in covariates by CRF were estimated using F tests. Logistic regression was used to determine the odds-ratios (ORs) and 95\% confidence interval (95\% CI) as an index of association between CRF and metabolic syndrome markers. In multivariate analyses, we initially adjusted for age and cigarette smoking. Analyses were performed with further adjustment for additional body fatness indices including percent body fat, body mass index (BMI), and waist-to-hip ratio (WHR) such that an independent role of CRF was tested in determining the prevalence of metabolic syndrome. The high CRF level was used as the reference category (OR 1.0). All statistical analyses were performed with SPSS-PC 11.0 for Windows (Chicago, IL, USA).

\section{RESULTS}

\section{Comparison of metabolic syndrome risk profiles across CRF levels}

Table 1 illustrates the comparison of body fatness and fitness and metabolic syndrome risk factors across CRF levels. In this study sample, there was a significant linear trend $(\mathrm{P}<0.001)$ in the overall prevalence of metabolic syndrome across CRF levels; $22.8 \%$ $(n=60)$ in the low CRF group, $7.1 \%(n=16)$ in the moderate CRF group, and $2.1 \%(n=9)$ in the high CRF group. Men with high CRF level were slightly younger than those with moderate $(\mathrm{P}<0.001)$ and low $(\mathrm{P}<0.001) \mathrm{CRF}$ levels, with no significant difference in age between the latter two groups. 
With respect to body fatness, there were inverse dose-response trends for lower body fatness across CRF levels such that the high CRF group had significantly lower BMI ( $\mathrm{P}<0.001$ \& $\mathrm{P}<0.001$, respectively), lower percent body fat $(\mathrm{P}<0.001 \& \mathrm{P}=0.080$, respectively), lower waist circumference ( $\mathrm{P}<0.001 \& \mathrm{P}<0.001$, respectively), and lower waist-to-hip ratio (WHR) $(\mathrm{P}<0.001 \& \mathrm{P}=0.001$, respectively) than the low and moderate CRF groups, and the moderate CRF group had even lower BMI $(\mathrm{P}<0.001)$, percent body fat $(\mathrm{P}<0.001)$, waist circumference $(\mathrm{P}<0.001)$, and WHR $(\mathrm{P}<0.001)$ than the low CRF group.

Similar and inverse trends for lower resting blood pressures across CRF levels were found such that the high CRF group had significantly lower systolic $(\mathrm{P}<0.001$ \& $\mathrm{P}=0.093$, respectively) and diastolic ( $\mathrm{P}<0.001 \& \mathrm{P}=0.010$, respectively) blood pressures than the low and moderate CRF groups, and the moderate CRF group had significantly lower values in systolic $(\mathrm{P}=0.003)$ and diastolic $(\mathrm{P}=0.001)$ blood pressures than the low CRF group.

With respect to blood lipids profile, there were inverse relationships between lipids and CRF levels; the high CRF group had a significantly lower value in TG than the low $(\mathrm{P}<0.001)$ and moderate $(\mathrm{P}<0.001) \mathrm{CRF}$ groups, and the moderate CRF group had a lower value in TG $(\mathrm{P}=0.015)$ than the low CRF group, with no group difference between the moderate and high CRF groups. A less clear, inverse trend in TC across the CRF levels was also found such that the high and moderate CRF groups had significantly lower TC concentrations ( $\mathrm{P}<0.001 \& \mathrm{P}=0.015$, respectively) than the low CRF group, with no group difference between the moderate and high CRF groups. No such group differences in HDLC were found across the CRF levels. 
Significant and inverse relationships of fasting values in glucose, insulin, and HOMAIR with CRF levels were found such that the high CRF group had significantly lower glucose ( $\mathrm{P}=0.008 \& \mathrm{P}=0.004$, respectively), insulin $(\mathrm{P}<0.001 \& \mathrm{P}=0.004$, respectively), and HOMA-IR $(\mathrm{P}<0.001 \& \mathrm{P}<0.001$, respectively) than the low and moderate $\mathrm{CRF}$ groups. Furthermore, the moderate CRF group had significantly lower insulin $(\mathrm{P}=0.015)$ and HOMA-IR $(\mathrm{P}=0.057)$ than the low $\mathrm{CRF}$ group.

In particular, we found a linear dose-response trend in nonsmoking habits across CRF levels; in the higher CRF levels there were more nonsmokers. In this study population, the proportions of subjects having metabolic syndrome were $2.1 \%, 7.1 \%$, and $22.8 \%$ in the high, moderate, and low CRF groups, respectively.

\section{CRF and Odds-Ratios for the Metabolic Syndrome}

Figure1 show ORs for having metabolic syndrome across CRF levels. After adjusting for age, the low and moderate CRF groups had odds of 14.28 (95\% CI, 6.87 to 29.66) $(\mathrm{P}<0.001)$ and 3.64 (95\% CI, 1.58 to 8.41$)(\mathrm{P}=0.002)$ for having metabolic syndrome than the high CRF group.

After adjusting for age and smoking, the low and moderate CRF groups had odds of 14.76 (95\% CI, 6.83 to 31.87) $(\mathrm{P}<0.001)$ and 3.88 (95\% CI, 1.62 to 9.26) $(\mathrm{P}=0.002)$ for having metabolic syndrome than the high CRF group.

After adjusting for age, smoking, and body fatness including BMI, \%BF, and WHR, the low and moderate CRF groups had odds of 4.64 (95\% CI, 2.00 to 10.79) $(\mathrm{P}<0.001)$ and 2.57 (95\% CI, 1.04 to 6.34) $(\mathrm{P}=0.039)$ for having metabolic syndrome than the high CRF group. 


\section{DISCUSSION}

To the best of our knowledge, this is the first report on low CRF as an independent risk factor for metabolic syndrome in young Korean men. From the current crosssectional study, we found that CRF is inversely and independently related to most of the metabolic syndrome risk factors in young Korean men, even after adjusting for potential compounding factors such as age, smoking, and body fatness, suggesting that maintaining moderate or higher CRF plays an important protective role in the development of metabolic syndrome and cardiovascular diseases including type 2 diabetes. The relationships we report here in Korean young adults have been consistently supported by the observations of previous cross-sectional $(2,12,16)$ and prospective $(2,14)$ studies.

A plausible mechanism that links low CRF to metabolic syndrome is high insulin resistance. This was demonstrated by Sato et al. (21), who showed a positive relationship between $\mathrm{VO}_{2}$ max with the rate of glucose metabolism. Ivy and Kuo (22) reported that individuals with low CRF have fewer glucose transporters compared with those who are more fit. In a recent study examining the effects of 6-month lifestyle intervention programs, Watkins et al. (23) reported that compared with control group, the exercise group experienced significant improvements in $\mathrm{VO}_{2} \mathrm{max}$, body fatness, and 2-hr postprandial insulin levels. In particular, fitness response analysis in their study showed a dose-response relationship between degree of improvement in $\mathrm{VO}_{2}$ max and degree of improvement in the 2-hr insulin response during oral glucose tolerance testing. The findings of the current study are also in an agreement with those previous 
findings in that men with high and moderate CRF levels had significantly lower fasting glucose, insulin, and HOMA-IR than men with low CRF level.

In addition, a recent cross-sectional study by Lee et al. (24) showed that Korean adults who did not exercise regularly had significantly larger waist circumference, higher TG, lower HDLC than those who exercised regularly. In the same study, the relative risk of developing metabolic syndrome was 1.7 times higher in the regular exercise group than in the non-exercise one, even after adjusting for age, sex, BMI, and other risk factors. Thus, a high prevalence of metabolic syndrome in spite of relatively lean body mass in the Korean adults might be explained by the thrifty gene hypothesis (25, 26). According to the hypothesis, poor CRF may be an intermediate phenotype of our sedentary and affluent society, in which thrifty genes have been chronically exposed to lack of physical activity and surplus energy, thereby contributing to the development of metabolic derangements such as abdominal obesity, insulin resistance, and type 2 diabetes $(27,28)$.

Although it is primarily influenced by individual differences in genetic make-up, CRF is a set of physiological attributes that are also substantially enhanced through participation in regular physical activity (29). Regular physical activity is a low-cost, safe therapy with minimal adverse side effects and favorable health benefits including a decrease in severity of each component of the metabolic syndrome (18). Pharmacological therapy for metabolic syndrome would require multiple agents, which increases costs and risks of side effects. Based on the findings of the current study, we believe that 1) health professionals, including clinicians and exercise scientists, should consider CRF in examining the relation between body fatness and metabolic syndrome 
in young Korean adults and 2) moderate or high CRF level, attained through increased physical activity, should be promoted in young Korean adults and considered to be one of the major preventive efforts against metabolic syndrome and its related chronic diseases in older Koreans.

\section{ACKNOWLEDGEMENTS}

This work was supported by a Korean Research Foundation Grant funded by the Korean Government (MOEHRD) (KRF-2005-042-G00025) and by the Samsung Research Fund of Sungkyunkwan University (2007-0316-000).

\section{DISCLOSURES}

We have no conflicts to disclose. 


\section{REFERENCES}

1. Kochanek KD, Smith BL. Deaths: preliminary data for 2002. Natl Vital Stat Rep 2004; 52: 1-47.

2. Carnethon MR, Gidding SS, Nehgme R, Sidney S, Jacobs DR Jr, Liu K. Cardiorespiratory fitness in young adulthood and the development of cardiovascular disease risk factors. JAMA 2003; 290: 3092-3100.

3. Huang KC, Lee LT, Chen CY, Sung PK. All-cause and cardiovascular disease mortality increased with metabolic syndrome in Taiwanese. Obesity (Silver Spring) 2008; 16(3): 684-689.

4. Isomaa B, Almgren P, Tuomi T, Forsén B, Lahti K, Nissén M, Taskinen MR, Groop L. Cardiovascular morbidity and mortality associated with the metabolic syndrome. Diabetes Care 2001; 24: 683-389.

5. Lakka HM, Laaksonen DE, Lakka TA, Niskanen LK, Kumpusalo E, Tuomilehto J, Salonen JT. The metabolic syndrome and total and cardiovascular disease mortality in middle-aged men. JAMA 2002; 288: 2709-2716.

6. Lee CD, Blair SN, Jackson AS. Cardiorespiratory fitness, body composition, and allcause and cardiovascular disease mortality in men. Am J Clin Nutr 1999; 69: 373-380.

7. Liese AD, Mayer-Davis EJ, Haffner SM. Development of the multiple metabolic syndrome: An epidemiologic perspective. Epidemiol Rev 1998; 20: 157-172.

8. Korean Ministry of Health and Welfare. The Korean National Health Nutrition Examination Survey 1998. Seoul: Korean Ministry of Health and Welfare 1999.

9. Korean Ministry of Health and Welfare. The Korean National Health Nutrition Examination Survey 2001. Korean Ministry of Health and Welfare: Seoul, 2002. p. $178-232$.

10. Expert Panel on Detection, Evaluation, and Treatment of High Blood Cholesterol in 
Adults. Executive summary of the third report of the National Cholesterol Education Program (NECP) expert panel on detection, evaluation, and treatment of high blood cholesterol in adults (Adult Treatment Panel III). JAMA 2001; 285: 2486-2497.

11. Kim D-J, Lee M-S, Kim K-W, Lee M-K. Insulin secretory dysfunction and insulin resistance in the pathogenesis of Korean type 2 diabetes mellitus. Metabolism 2001; 50: 590-593.

12. Boulé NG, Bouchard C, Tremblay A. Physical fitness and the metabolic syndrome in adults from the Quebec Family Study 2005. Can J Appl Physiol 2005; 30: 140-156.

13. Lakka TA, Laaksonen DE, Lakka HM, Männikkö N, Niskanen LK, Rauramaa R, Salonen JT. Sedentary lifestyle, poor cardiorespiratory fitness, and the metabolic syndrome. Med Sci Sports Exerc 2003; 35: 1279-1286.

14. LaMonte MJ, Ainsworth BE, Durstine JL. Influence of cardiorespiratory fitness on the association between C-reactive protein and metabolic syndrome prevalence in racially diverse women. J Womens Health (Larchmt) 2005; 14: 233-239.

15. Wareham NJ, Hennings SJ, Byrne CD, Hales CN, Prentice AM, Day NE. A quantitative analysis of the relationship between habitual energy expenditure, fitness and the metabolic cardiovascular syndrome. Br J Nutr 1999; 80: 235-241.

16. Whaley MH, Kampert JB, Kohl HW 3rd, Blair SN. Physical fitness and clustering of risk factors associated with the metabolic syndrome. Med Sci Sports Exerc 1999; 31: 287-293.

17. Zhu S, St-Onge MP, Heshka S, Heymsfield SB. Lifestyle behaviors associated with lower risk of having the metabolic syndrome. Metabolism 2004; 53: 1503-1511. 
18. American College of Sports Medicine. ASCM's guidelines for exercise testing and prescription. 7th ed. Philadelphia, PA: Lippinocott Williams \& Wilkins, 2006, p $237-240$.

19. Matthews DR, Hosker JP, Rudenski AS, Naylor BA, Treacher DF, Turner RC. Ho meostasis model assessment: insulin resistance and beta cell function from fasting plasma glucose and insulin concentrations in man. Diabetologia 1985; 28: 412-419

20. Steering Committee. The Asia-Pacific perspective: Redefining obesity and its treatment. Melbourne: International Diabetes Institute 2000.

21. Sato Y, Iguchi A, Sakamoto N. Biochemical determination of training effects using insulin clamp technique. Horm Metab Res 1984; 16: 483-386.

22. Ivy JL, Kuo CH. Regulation of GLUT4 protein and glycogen synthase during muscle glycogen synthesis after exercise. Acta Physiol Scand 1998; 162: 295-304.

23. Watkins LL, Sherwood A, Feinglos M, Hinderliter A, Babyak M, Gullette E, Waugh R, Blumenthal JA. Effects of exercise and weight loss on cardiac risk factors associated with syndrome X. Arch Intern Med 2003; 163: 1889-1895.

24. Lee W-Y, Jung C-H, Park J-S, Rhee E-J, Kim S-W. Effects of smoking, alcohol, exercise, education, and family history on the metabolic syndrome as defined by the ATP III. Diabetes Res Clin Pract 2005;67:70-77.

25. Groop L. Genetics of the metabolic syndrome. Br J Nutr 2000;83:S39-S48.

26. Neel JV. Diabetes mellitus: a 'thrifty' genotype rendered detrimental by ‘progress’? Am J Hum Genet 1962; 14: 353-362.

27. Chakravarthy MV, Booth FW. Eating, exercise, and "thrifty" genotypes: 
connecting the dots toward an evolutionary understanding of modern chronic diseases. J Appl Physiol 2004; 96: 3-10.

28. Speakman JR. Thrifty genes for obesity and the metabolic syndrome - time to call off the search? Diab Vasc Dis Res 2006; 3: 7-11.

29. Bouchard C, Perusse L. Heredity, activity levels, fitness, and health. In: Bouchard C, Shephard RJ, Stephens T, eds. Physical Activity, Fitness, and Health: International Proceedings and Consensus Statement. Champaign, III: Human Kinetics Publishers; 1994. 
TABLE 1. Description of the study participants.

\begin{tabular}{|c|c|c|c|c|}
\hline & LOW CRF & MOD CRF & HIGH CRF & $\mathrm{P}$ value for linear trend \\
\hline $\mathrm{N}$ & 263 & 224 & 422 & \\
\hline Age (years) & $24.5 \pm 2.2$ & $24.1 \pm 2.5$ & $23.4 \pm 2.2$ & $<0.001$ \\
\hline Body mass (kg) & $68.1 \pm 8.0$ & $69.7 \pm 8.3$ & $74.7 \pm 11.3$ & $<0.001$ \\
\hline BMI $\left(\mathrm{kg} / \mathrm{m}^{2}\right)$ & $24.3 \pm 3.5$ & $22.7 \pm 2.4$ & $22.3 \pm 2.1$ & $<0.001$ \\
\hline Body fat (\%) & $22.6 \pm 5.6$ & $19.6 \pm 4.3$ & $17.9 \pm 4.3$ & $<0.001$ \\
\hline Waist circumference $(\mathrm{cm})$ & $86.5 \pm 8.9$ & $81.7 \pm 6.6$ & $79.5 \pm 5.9$ & $<0.001$ \\
\hline Waist-to-hip ratio (WHR) & $0.87 \pm 0.05$ & $0.85 \pm 0.05$ & $0.83 \pm 0.04$ & $<0.001$ \\
\hline Systolic blood pressure (mmHg) & $124.3 \pm 13.1$ & $120.9 \pm 12.3$ & $119.2 \pm 11.6$ & $<0.001$ \\
\hline Diastolic blood pressure (mmHg) & $72.9 \pm 9.2$ & $70.3 \pm 8.4$ & $68.6 \pm 7.7$ & $<0.001$ \\
\hline Total cholesterol (mg/dl) & $178.4 \pm 34.8$ & $171.1 \pm 32.2$ & $168.1 \pm 30.1$ & $<0.001$ \\
\hline Triglycerides (mg/dl) & $136.1 \pm 69.8$ & $111.1 \pm 51.7$ & $95.9 \pm 42.6$ & $<0.001$ \\
\hline HDL cholesterol (mg/dl) & $45.5 \pm 13.2$ & $45.4 \pm 13.7$ & $46.1 \pm 11.9$ & 0.757 \\
\hline
\end{tabular}




$\begin{array}{lcccc}\text { Fasting glucose }(\mathrm{mg} / \mathrm{dl}) & 98.5 \pm 10.8 & 98.6 \pm 11.6 & 94.9 \pm 11.3 & 0.003 \\ \text { Fasting insulin }(\mu \mathrm{U} / \mathrm{ml}) & 8.31 \pm 6.29 & 6.90 \pm 4.46 & 5.50 \pm 3.26 & <0.001 \\ \text { HOMA-IR } & 2.04 \pm 1.57 & 1.75 \pm 1.33 & 1.28 \pm 0.77 & <0.001 \\ \text { Smoking }(\%) & 40.5 / 12.5 / 46.1 & 31.3 / 12.4 / 55.7 & 25.2 / 15.4 / 58.7 & \text { current-/past-/non-smokers } \\ \text { Metabolic syndrome } & (22.8 \%, \mathrm{n}=60) & (7.1 \%, \mathrm{n}=16) & (2.1 \%, \mathrm{n}=9)\end{array}$

Data are mean \pm SD. BMI: body mass index; Triglycerides, insulin, and HOMA index (HOMA-IR) were log10 transformed for the normalization of distribution prior to statistical analyses, and the raw data were presented in the table. 


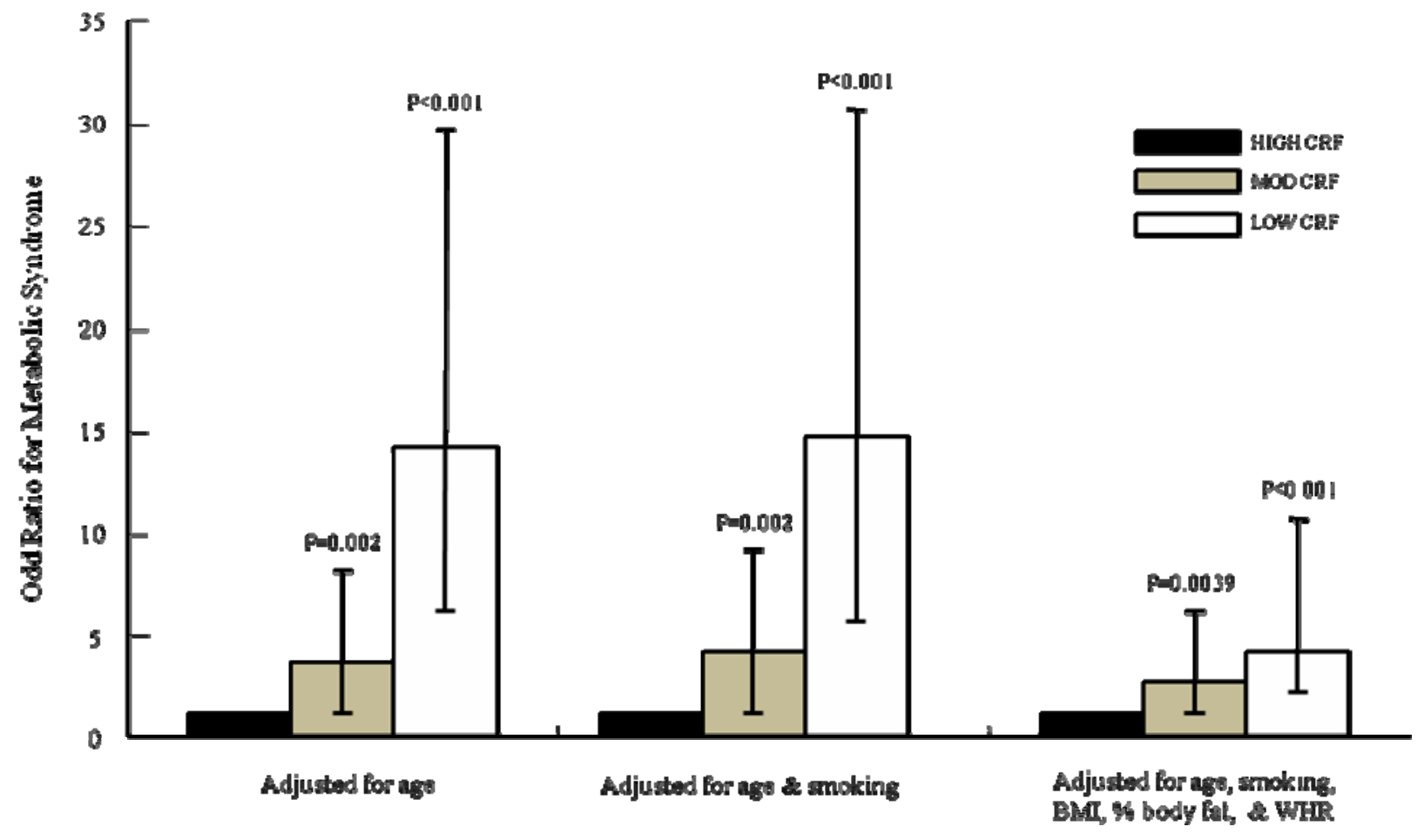

Figure 1. Odds-ratios and 95\% confidence intervals for metabolic syndrome after adjusting for age, smoking, BMI, \% body fat, and WHR. P values are for the comparison tests with the high CRF group. 